\title{
Phase diagram of the restricted solid-on-solid model coupled to the Ising model
}

\author{
Sooyeul Lee and Koo-Chul Lee \\ Department of Physics and Center for Theoretical Physics \\ Seoul National University \\ Seoul 151-742, Korea \\ J. M. Kosterlitz \\ Department of Physics \\ Brown University \\ Providence, Rhode Island 02912
}

\begin{abstract}
We study the phase transitions of a restricted solid-on-solid model coupled to an Ising model, which can be derived from the coupled $X Y$-Ising model. There are two kinds of phase transition lines. One is a Ising transition line and the other is surface roughening transition line. The latter is a KT transition line from the viewpoint of the $X Y$ model. Using a microcanonical Monte Carlo technique, we obtain a very accurate two dimensional phase diagram. The two transition lines are separate in all the parameter space we study. This result is strong evidence that the fully frustrated $X Y$ model orders by two separate transitions and that roughening and reconstruction transitions of crystal surfaces occur separately.
\end{abstract}

PACS numbers:64.60.-i, 64.60.C, 75.40.Mg

Typeset using REVTEX 


\section{INTRODUCTION}

Since the pioneering work of Teitel and Jayaprakash 1 , two dimensional (2D) fully frustrated $X Y(\mathrm{FF} X Y)$ models have been intensively studied over the last decade, both analytically and numerically.2 8 This model has a continuous $U(1)$ symmetry associated with global spin rotations and also a discrete $Z(2)$ symmetry because of the double degeneracy of the ground state vortex configurations. At sufficiently low temperature $(T)$ the system will be completely ordered with true long range order in the Ising order parameter characterizing the vortex lattice and algebraic decay of correlations of the $X Y$ order parameter. As $T$ is increased, the system will disorder by one of two possible scenarios. One is the simultaneous loss of both $X Y$ and Ising order and the other is a two stage process in which $X Y$ order is destroyed at a lower temperature than the Ising order. Since the original paper on this model, 1 an enormous effort has gone into trying to clarify the nature of the phase transition(s) of this model and several related models believed to be in the same universality class have been studied numerically. 38 However, the nature of the transition(s) in the FF $X Y$ model and its generalizations is still somewhat controversial. Monte Carlo (MC) simulations of the Coulomb gas with half integer charges 3 : 14 generally show a vortex lattice melting transition and a KT-like transition at slightly different temperatures which is consistent with the second scenario. However, the melting transition is expected to be in the Ising universality class but the simulations yield exponents which are inconsistent with the Ising values. Moreover, at the KT-like transition the discontinuity in the helicity modulus is much larger than the universal value of $2 / \pi$. Yet another model is the coupled $X Y$-Ising model ln $^{\mathrm{B}}$ wich is a coarse-grained version of the FF XY model. It was suggested that this model displayed both possible scenarios depending on the parameters of the model. Recent studies 6 FFXY model with a nearest neighbor cosine interaction show that the disordering is by the second scenario with a KT transition followed by an Ising transition at a slightly higher temperature. From a theoretical point of view, the FF $X Y$ model may have separate $X Y$ and Ising transitions if the critical exponents of the vortex lattice melting are consistent 
with the 2D Ising values and the jump in the helicity modulus at the $X Y$ transition has the universal value of $2 / \pi$ but most numerical studies are not consistent with these. However, in his recent numerical study, Olssont pointed out that, for conventional finite size scaling to hold, it is essential that the system size $L$ is much larger than any other length scale. Since the vortex lattice melting temperature $T_{c \mathrm{I}}$ is only slightly larger than $T_{\mathrm{KT}}$, the relevant length is $\xi_{+}\left(T_{c \mathrm{I}}\right)$, the $X Y$ correlation length at the lattice melting temperature. As is well known, this remains rather large quite far above $T_{\mathrm{KT}}$ and, by taking this into account, a good fit to the double transition scenario was obtained with a conventional KT transition followed by an Ising transition.

Whatever the precise nature of the transitions in any model, an essential first step is to determine whether the system has two separate transitions or a single transition. It is of some interest from the point of view of conformal field theories to find a non-trivial model in which the central charge $c>1$. Recently, a related class of models for transitions on crystal surfaces with competing reconstruction (Ising) and roughening $(X Y)$ degrees of freedom has been studied 11, 12. Den Nijs has studied competition between surface roughening and reconstruction and tentatively concluded that the roughening and deconstruction transition lines meet and finally merge together into a single roughening induced simultaneous reconstruction transition line.1.1 On the other hand, Carlon in a recent study of the staggered six vertex model, 12 has suggested that the two transition lines never merge but become very close to each other which is strong evidence for two separate transition in the FFXY model. In order to clarify whether the Ising and $X Y$ transition lines merge we introduce the restricted solid-on-solid (RSOS) model coupled to the Ising model. This RSOS-Ising model can be derived from the $X Y$-Ising model by making a duality transformation on the $X Y$ degrees of freedom which introduces integer height variables on the dual lattice. The resulting RSOS-Ising model has both Ising and height $(X Y)$ degrees of freedom and, for this reason, can be considered to have the same features as the staggered six vertex model and reconstructed surface models.

We investigate the RSOS-Ising system by a rejection free microcanonical MC technique 3 
which is appropriate to this system and also highly accurate. We have obtained an accurate phase diagram in a two parameter space and some critical exponents. As a result we have found there are Ising and roughening critical lines which never merge in all parameter space. This result is strong evidence against the single transition scenario in the fully frustrated $X Y$ model.

In the following section we give a derivation of the RSOS-Ising model from the coupled $X Y$-Ising system. In section III we present a brief explanation of the MC procedure ${ }^{13}$ as adapted for this system, methods of data analysis and MC results. Finally we present our conclusions.

\section{RESTRICTED SOLID-ON-SOLID MODEL COUPLED TO ISING MODEL}

From renormalization group and universality arguments the isotropic FF $X Y$ model can be described by a coupled $X Y$-Ising model of the form

$$
-\beta H=A_{0} \sum_{\langle i, j\rangle}\left(1+\sigma_{i} \sigma_{j}\right) \cos \left(\theta_{i}-\theta_{j}\right)+C_{0} \sum_{\langle i, j\rangle} \sigma_{i} \sigma_{j},
$$

where $\theta_{i}$ is the phase of the continuous spin at $i$ th site and $\sigma_{i}= \pm 1$ describes the chirality. The physical range of the phenomenological parameters is $A_{0}>0$ and $A_{0}+C_{0}>0$. This

model has been intensively studied by Lee et al . (Ising) degrees of freedom $\sigma_{i}$, they found a line of continuous transitions with a segment of non-Ising like critical exponents, in particular $\nu_{I} \approx 0.8<1$ and $\eta_{I}>1 / 4$. Based on this, they argued that the Ising and $X Y$ critical lines must have merged into a single line of simultaneous ordering of both degrees of freedom. However, they made no explicit study of the $X Y$ degree of freedom for technical reasons, which weakens their argument. To simultaneously study the $X Y$ and Ising critical lines, we make a duality transformation 4 on the $X Y$ variables of Eq.(1) to obtain a restricted solid-on-solid model coupled to an Ising model, in which only discrete variables are involved, as described below.

The partition function of the $X Y$-Ising model of Eq.(1) reads 


$$
Z=\sum_{\{\sigma\}} \exp \left[C_{0} \sum_{\langle i, j\rangle} \sigma_{i} \sigma_{j}\right] \int_{0}^{2 \pi} \cdots \int_{0}^{2 \pi} \prod_{k=1}^{N} \mathrm{~d} \theta_{k} \prod_{\langle i, j\rangle} \exp \left[K_{i j} \cos \left(\theta_{i}-\theta_{j}\right)\right],
$$

where $\{\sigma\}$ denotes the sum over all configurations of Ising spins, $N$ is the number of lattice sites and $K_{i j}=A_{0}\left(1+\sigma_{i} \sigma_{j}\right)$. Using the periodicity of the integrand in $\theta_{i j} \equiv \theta_{i}-\theta_{j}$ we have

$$
\exp \left[K_{i j} \cos \left(\theta_{i}-\theta_{j}\right)\right]=\sum_{n_{i j}=-\infty}^{\infty} \exp \left[i n_{i j} \theta_{i j}\right] \exp \left[\tilde{V}\left(n_{i j}\right)\right]
$$

where $\exp \left[\tilde{V}\left(n_{i j}\right)\right]$ is a Fourier component of $\exp \left[K_{i j} \cos \left(\theta_{i}-\theta_{j}\right)\right]$. This yields

$$
Z=\sum_{\{\sigma\}} \exp \left[C_{0} \sum_{\langle i, j\rangle} \sigma_{i} \sigma_{j}\right] \int_{0}^{2 \pi} \cdots \int_{0}^{2 \pi} \prod_{k=1}^{N} \mathrm{~d} \theta_{k} \prod_{\langle i, j\rangle} \sum_{n_{i j}=-\infty}^{\infty} \exp \left[i n_{i j} \theta_{i j}+\tilde{V}\left(n_{i j}\right)\right] .
$$

The integrations over the $\theta_{k}$ can be easily done and, noting that $n_{i j}=-n_{j i}$, give $N$ constraints on the $2 N$ integers $n_{i j}$ so the partition function has the form

$$
Z=\sum_{\{\sigma\}} \exp \left[C_{0} \sum_{\langle i, j\rangle} \sigma_{i} \sigma_{j}\right] \sum_{\{n\}}\left[\prod_{i} \Delta(i)\right] \prod_{\langle k, l\rangle} \exp \left[\tilde{V}\left(n_{k l}\right)\right]
$$

where the $\Delta(i)$ denote the constraints at site $i$. For example, the case as shown in Fig. 1 gives $\Delta(0)=\delta_{n_{01}+n_{02}, n_{30}+n_{40}}$. The $N$ constraints are automatically satisfied by introducing the integer height variables $h_{I}$ on the dual lattice shown in Fig. 1 as

$$
\begin{aligned}
& n_{01}=h_{1}-h_{2}, \quad n_{02}=h_{2}-h_{3} \\
& n_{30}=h_{4}-h_{3}, \quad n_{40}=h_{1}-h_{4} .
\end{aligned}
$$

In terms of the heights $h_{I}$ the partition function has the apparently simple form

$$
Z=\sum_{\{\sigma\}} \exp \left[C_{0} \sum_{\langle i, j\rangle} \sigma_{i} \sigma_{j}\right] \sum_{\{h\}} \exp \left[\sum_{\langle I, J\rangle} \tilde{V}\left(h_{I}-h_{J}\right)\right]
$$

where $I$ and $J$ are dual lattice sites and $-\infty<h_{I}<\infty$. To this point everything is exact but Eq.(6) is not suitable for simulation because of the infinite range of integer height differences $h_{I}-h_{J}$. The Fourier component is

$$
\exp \left[\tilde{V}\left(h_{I}-h_{J}\right)\right]=\frac{1}{2 \pi} \int_{0}^{2 \pi} \exp \left[-i\left(h_{I}-h_{J}\right) \theta_{i j}\right] \exp \left[A_{0}\left(1+\sigma_{i} \sigma_{j}\right) \cos \theta_{i j}\right] \mathrm{d} \theta_{i j}
$$


where the nearest neighbor bonds $I J$ on the dual lattice and $i j$ on the original lattice cross each other. If $\sigma_{i} \sigma_{j}=-1$, the Fourier component $\exp [\tilde{V}(h)]=\delta_{h, 0}$. A step in $h_{I}$ cannot cross an Ising domain wall. When $\sigma_{i} \sigma_{j}=1$ Eq. (17) becomes $I_{h}\left(2 A_{0}\right)$, where $I_{h}$ is a Bessel function of order $h$ with imaginary argument and $h=h_{I}-h_{J}$ and $I_{h}(0)=\delta_{h, 0}$. From the asymptotic behavior of the Bessel functions we have $I_{h}\left(2 A_{0}\right) / I_{0}\left(2 A_{0}\right) \approx \exp \left(-h^{2} / 4 A_{0}\right)$, which decays rapidly as $h$ increases if the inverse temperature $A_{0}$ is not too large. Therefore, it is a reasonable approximation to keep only the contributions from $h_{I}-h_{J}=0, \pm 1$ when $\sigma_{i} \sigma_{j}=1$

In view of these arguments, an effective Hamiltonian for the system is

$$
-\beta H=C \sum_{\langle i, j\rangle} \sigma_{i} \sigma_{j}-A \sum_{\langle I, J\rangle}\left|h_{I}-h_{J}\right|
$$

where $\left(h_{I}-h_{J}\right)=0, \pm 1$ if the corresponding $\sigma_{i} \sigma_{j}=1$ and $h_{I}=h_{J}$ otherwise. By comparing the Boltzmann weights derived from the Hamiltonians (11) and (8) we obtain the relations $\exp (-A)=I_{1}\left(2 A_{0}\right) / I_{0}\left(2 A_{0}\right)$ and $\exp (2 C)=\exp \left(2 C_{0}\right) I_{0}\left(2 A_{0}\right) . A$ is inversely related to $A_{0}$ and $C$ is a linear sum of $C_{0}$ and $A_{0}$. Eq. (8) is the Hamiltonian for a RSOS-Ising model and the heights $h_{I}$ and Ising spins $\sigma_{i}$ are coupled via the constraint that a step in $h$ is forbidden to cross a domain wall in $\sigma$.

\section{MONTE CARLO ANALYSIS}

Due to the restrictions on the spin and height variables discussed in the last section, some spin-height configurations are not allowed which we call forbidden configurations. In our MC procedure, we select a spin or height variable randomly and update the configuration by a trial move. The trial move for the spin variable $\sigma$ is a simple flipping, i.e., $\sigma \rightarrow-\sigma$. For the height variable $h$, the trial move is either raise or lower the selected height by 1 unit,

i.e., $h \rightarrow h \pm 1$. If the attempted move takes the system to a forbidden configuration, we call the move forbidden and otherwise we call the move allowed. The number of forbidden moves changes according to the configuration of the neighboring spins and heights. For 
accuracy and efficiency, we use the microcanonical MC method 13 introduced by one of the authors to study thermodynamic functions of the system in the $2 \mathrm{D}$ parameter space $(A, C)$. We further apply a rejection-free technique to speed up the MC procedure.13 To apply the microcanonical MC method to the RSOS-Ising model we first introduce an energy-like quantity

$$
E=E_{\mathrm{I}}+E_{\mathrm{R}}
$$

where $E_{\mathrm{I}}=-\sum_{\langle i, j\rangle} \sigma_{i} \sigma_{j}$ and $E_{\mathrm{R}}=2 \sum_{\langle I, J\rangle}\left|h_{I}-h_{J}\right|$. The factor 2 in $E_{\mathrm{R}}$ is introduced for convenience to make the minimum energy changes $\Delta E_{\mathrm{R}}=\Delta E_{\mathrm{I}}=4$ for both $E_{\mathrm{R}}$ and $E_{\mathrm{I}}$. The quantities to be computed are the number of configurations $\tilde{\omega}\left(E, E_{\mathrm{I}}\right)$ and the microcanonical average $\tilde{Q}\left(E, E_{\mathrm{I}}\right)$ of a thermodynamic quantity $Q$ at a set of values of $E$ and $E_{\mathrm{I}}$.

Since the rejection free MC technique 13 is not well known, we present a brief account of the microcanonical MC method as adapted to the RSOS-Ising system. We set up a random walk through a configuration space restricted to a small energy square in the $\left(E, E_{\mathrm{I}}\right)$ domain given by

$$
\begin{gathered}
E^{(i)}-q \Delta E / 2 \leq E \leq E^{(i+1)}+q \Delta E / 2 \\
E_{\mathrm{I}}^{(j)}-q \Delta E_{\mathrm{I}} / 2 \leq E_{\mathrm{I}} \leq E_{\mathrm{I}}^{(j+1)}+q \Delta E_{\mathrm{I}} / 2
\end{gathered}
$$

where $\Delta E=4$ is the minimum energy separation in $E, q$ is the coordination number of the lattice, $E^{(i+1)}=E^{(i)}+\Delta E$ and $E_{\mathrm{I}}^{(j+1)}=E_{\mathrm{I}}^{(j)}+\Delta E_{\mathrm{I}} \cdot q \Delta E / 2$ and $q \Delta E_{\mathrm{I}} / 2$ are the maximum energy changes in $E$ and $E_{\mathrm{I}}$ when a spin or height variable is updated to a new state. The size of the square given by Eqs. (10) and (11) is chosen to make any allowed move keep the new configuration within this square if the current configuration is in the innermost square, a configuration whose $E$ and $E_{\mathrm{I}}$ values belonging to one of four sets, $\left(E^{(i)}, E_{\mathrm{I}}^{(j)}\right)$, $\left(E^{(i+1)}, E_{\mathrm{I}}^{(j)}\right),\left(E^{(i)}, E_{\mathrm{I}}^{(j+1)}\right)$, and $\left(E^{(i+1)}, E_{\mathrm{I}}^{(j+1)}\right)$.

Since there are $N$ candidates for the trial spin move and $2 N$ candidates for the trial height move, there is a total of $\tilde{N} \equiv 3 N$ of candidates for the trial move. Then out of this $\tilde{N}$, there are $\tilde{N}_{\text {alwd }}$ allowed and $\tilde{N}_{\text {fbdn }}=\tilde{N}-\tilde{N}_{\text {alwd }}$ forbidden moves. We tabulate both 
allowed and forbidden moves in the same look-up table. The allowed moves are divided into two groups, one consisting of acceptable moves which keep the walker within the predefined energy domain given by Eqs. (10) and (11) and the other of rejectable moves which take the walker outside this square. If the walker is initially at a configuration belonging to the innermost square, all allowed moves are acceptable. On the other hand, if the walker is not at configuration belonging to the innermost four energy values, then some allowed moves are acceptable and some are rejectable since some moves will take the random walker outside the square of Eqs. (10) and (11) as the largest energy changes in $E$ and $E_{\mathrm{I}}$ are $q \Delta E / 2$ and $q \Delta E_{\mathrm{I}} / 2$

Whenever the walker visits one of the configuration belonging to the innermost square we count the number of visit and take samples of $Q$ 's. Next we select a trial move randomly from all possible $\tilde{N}$ moves. If the selected move is an allowed one, we update the configuration since it is always acceptable. If the selected move is forbidden we discard the move but count the current configuration once more and sample again for the same configuration to satisfy the condition of detailed balance. This procedure is equivalent to assigning the correct weight to the sampling configurations. When the walker is at a configuration not belonging to the innermost four energy values, we make a random selection of trial move from the group of acceptable moves only and let the walker move to a new configuration without wasting computing time. This is possible since we are not taking data at this configuration. In this way we can estimate the number of visits for the innermost four energy configurations, which give relative ratios $\tilde{\omega}\left(E^{(i)}, E_{\mathrm{I}}^{(j+1)}\right) / \tilde{\omega}\left(E^{(i)}, E_{\mathrm{I}}^{(j)}\right)$ and $\tilde{\omega}\left(E^{(i+1)}, E_{\mathrm{I}}^{(j)}\right) / \tilde{\omega}\left(E^{(i)}, E_{\mathrm{I}}^{(j)}\right)$ for all $i$ and $j$. The sampled data for $Q$ will furnish microcanonical averages $\tilde{Q}\left(E, E_{\mathrm{I}}\right)$. Since the exact number of configurations $\tilde{\omega}\left(E, E_{\mathrm{I}}\right)$ for small values of $E$ and $E_{\mathrm{I}}$ are easily computed, we can recursively estimate all $\tilde{\omega}\left(E, E_{\mathrm{I}}\right)$ 's from these. To obtain more accurate data we use a two-step MC simulation. First we accurately estimate $\tilde{\omega}\left(E^{(i+1)}\right) / \tilde{\omega}\left(E^{(i)}\right)$ for all $i$, where $\tilde{\omega}\left(E^{(i)}\right)=\sum_{E_{\mathrm{I}}} \tilde{\omega}\left(E^{(i)}, E_{\mathrm{I}}\right)$. We then estimate $\tilde{\omega}\left(E^{(i)}, E_{\mathrm{I}}^{(j+1)}\right) / \tilde{\omega}\left(E^{(i)}, E_{\mathrm{I}}^{(j)}\right)$ for all $j$. Now we can easily estimate all $\tilde{\omega}\left(E, E_{\mathrm{I}}\right)$ consistently. The microcanonical average $\tilde{Q}\left(E^{(i)}, E_{\mathrm{I}}^{(j)}\right)$ is also estimated directly from data taken during this MC process. To check the validity of our 
procedure we compare the estimated $\tilde{\omega}\left(E_{\mathrm{R}}, 0\right)$ and $\tilde{\omega}\left(E_{\mathrm{I}}, E_{\mathrm{I}}\right)$ for all system sizes with those of the pure RSOS model and those of the pure Ising model respectively. We calculated the number of configurations for the pure RSOS model independently and the those of the pure Ising model are well known 13 . Both of them agree well with each other to within expected statistical deviations.

The canonical average of any thermodynamic quantity $Q$ is obtained by

$$
\langle Q\rangle=\frac{\sum_{E E_{\mathrm{I}} M_{\mathrm{R}} M_{\mathrm{I}}} Q\left(E, E_{\mathrm{I}}, M_{\mathrm{R}}, M_{\mathrm{I}}\right) \omega\left(E, E_{\mathrm{I}}, M_{\mathrm{R}}, M_{\mathrm{I}}\right) \exp \left(-C E_{\mathrm{I}}-A E_{\mathrm{R}} / 2\right)}{\sum_{E E_{\mathrm{I}} M_{\mathrm{R}} M_{\mathrm{I}}} \omega\left(E, E_{\mathrm{I}}, M_{\mathrm{R}}, M_{\mathrm{I}}\right) \exp \left(-C E_{\mathrm{I}}-A E_{\mathrm{R}} / 2\right)},
$$

where $M_{\mathrm{I}}=L^{-2}\left|\sum_{i} \sigma_{i}\right|$ is the Ising magnetization, $M_{\mathrm{R}}$ is a RSOS magnetization to be defined in Eq.(14) below and $Q\left(E, E_{\mathrm{I}}, M_{\mathrm{R}}, M_{\mathrm{I}}\right)$ and $\omega\left(E, E_{\mathrm{I}}, M_{\mathrm{R}}, M_{\mathrm{I}}\right)$ are the microcanonical values of the thermodynamic quantity $Q$ and the number of configurations for given ( $\left.E, E_{\mathrm{I}}, M_{\mathrm{R}}, M_{\mathrm{I}}\right)$, respectively. In Eq.(12), the exponent of the Boltzmann weight factor does not involve any magnetization. We thus estimate only $\tilde{\omega}\left(E, E_{\mathrm{I}}\right) \equiv \sum_{M_{\mathrm{R}} M_{\mathrm{I}}} \omega\left(E, E_{\mathrm{I}}, M_{\mathrm{R}}, M_{\mathrm{I}}\right)$ and $\tilde{Q}\left(E, E_{\mathrm{I}}\right) \equiv \sum_{M_{\mathrm{R}} M_{\mathrm{I}}} \omega\left(E, E_{\mathrm{I}}, M_{\mathrm{R}}, M_{\mathrm{I}}\right) Q\left(E, E_{\mathrm{I}}, M_{\mathrm{R}}, M_{\mathrm{I}}\right) / \tilde{\omega}\left(E, E_{\mathrm{I}}\right)$. If $Q$ depends only on $E$ and $E_{\mathrm{I}}$, as in the case of the specific heat, we need only $\tilde{\omega}\left(E, E_{\mathrm{I}}\right)$ since $\tilde{Q}\left(E, E_{\mathrm{I}}\right)=$ $Q\left(E, E_{\mathrm{I}}\right)$. Otherwise we need to calculate both $\tilde{\omega}\left(E, E_{\mathrm{I}}\right)$ and $\tilde{Q}\left(E, E_{\mathrm{I}}\right)$. The canonical average of $Q$ can be written as

$$
\langle Q\rangle=\frac{\sum_{E E_{\mathrm{I}}} \tilde{Q}\left(E, E_{\mathrm{I}}\right) \tilde{\omega}\left(E, E_{\mathrm{I}}\right) \exp \left(-C E_{\mathrm{I}}-A E_{\mathrm{R}} / 2\right)}{\sum_{E E_{\mathrm{I}}} \tilde{\omega}\left(E, E_{\mathrm{I}}\right) \exp \left(-C E_{\mathrm{I}}-A E_{\mathrm{R}} / 2\right)} .
$$

All thermodynamic quantities we use in this paper depend only on the energies $E$ and $E_{\mathrm{I}}$ or only on magnetizations $M_{\mathrm{R}}$ and $M_{\mathrm{I}}$. For example, $Q\left(E, E_{\mathrm{I}}, M_{\mathrm{R}}, M_{\mathrm{I}}\right)=E$ for the total energy, $Q=M_{\mathrm{I}}$ for the Ising magnetization and $Q=\delta_{M_{\mathrm{I}}, M}$ for the magnetization probability distribution $P_{L}(M)$. In all cases, samplings are performed in the $2 \mathrm{D}$ plane of $\left(E, E_{\mathrm{I}}\right)$. The number of possible $E$ and $E_{\mathrm{I}}$ is proportional to $L^{2}$ and the simulation time increases as $L^{4}$ where $L$ is the lattice size. This makes it difficult to simulate systems of large size. Nevertheless, once $\tilde{Q}\left(E, E_{\mathrm{I}}\right)$ and $\tilde{\omega}\left(E, E_{\mathrm{I}}\right)$ are estimated, the canonical average $\langle Q\rangle$ can be easily calculated for all $A$ and $C$ from Eq. (13).

As the specific heat of the 2D $X Y$ model has no diverging peak as $L \rightarrow \infty$, the RSOS part of the specific heat may also not diverge which implies the transition temperature 
may not be easy to determine accurately. For the 2D $X Y$ model, which has vanishing magnetization in the bulk limit for any finite temperature, the helicity modulus, or stiffness constant which is a measure of the quasi long-range order of the order parameter describing the continuous $U(1)$ symmetry and the superfluid density of a Bose superfluidet, may be used to determine the $X Y$ transition temperature from the finite size scaling form 10 at the $\mathrm{KT}$ point. The analogous quantity in the RSOS model is the free energy of a step in the height which has the finite size scaling form $16 F_{s}(L)=\pi / 4+1 /(A+B \ln L)$ at the roughening temperature. However, the computation of this requires the partition function with two different boundary conditions which is time consuming. As an alternative method, we know that the roughening transition in the RSOS model is one between flat and rough phases. We introduce an order parameter, analogous to the magnetization of an Ising model, $M_{\mathrm{R}}$, which measures the flatness of the RSOS system, as

$$
M_{\mathrm{R}}=L^{-2} \sum_{I} m_{\mathrm{R}}\left(h_{I}\right)
$$

where $m_{\mathrm{R}}\left(h_{I}\right)=1$ if the height $h_{I}$ is equal to the majority height and $m_{\mathrm{R}}=0$ otherwise. It is clear from this definition that $M_{\mathrm{R}}=1$ for a completely flat phase and vanishes in a rough phase in the thermodynamic limit. In Figs. $2(\mathrm{a})$ and $2(\mathrm{~b})$ we plot $M_{\mathrm{R}}$ and $M_{\mathrm{I}}$ in the parameter space $(A, C)$ for lattice size $L=22$. It is easily seen from the figures that there are three different phases depending on the parameters $A$ and $C$ : Ising ordered rough (IOR), Ising ordered flat (IOF) and Ising disordered flat (DOF). The IOR and DOF phases are separated by the IOF phase in the low temperature region though it is not clear whether, in the region of small $A$ and $C$, the two phases are also separated or not. The corresponding phases of the $X Y$-Ising model of Eq.(1) are $X Y$ and Ising order (IOR), $X Y$ disorder and Ising order (IOF) and $X Y$ and Ising disorder (DOF). It is interesting to note that the fourth phase of $X Y$ order and Ising disorder, corresponding to an Ising disordered rough phase of the RSOS-Ising model, does not exist.

We now examine the behavior of the specific heat. For simplicity, we consider $C_{\mathrm{R}}$ and $C_{\mathrm{I}}$ corresponding to the RSOS and Ising specific heats, respectively. These are defined as 
$L^{2} C_{\mathrm{S}}=-D^{2} \partial\left\langle E_{\mathrm{S}}\right\rangle / \partial D$, where $D, \mathrm{~S}=A, \mathrm{R}$ and $C, \mathrm{I}$ respectively. In Figs. 3(a) and 3(b) we plot the RSOS and Ising specific heats for lattice size $L=22$. We have obtained the specific heats for several lattice sizes up to $L=22$. The RSOS specific heat ridge parallel to the $C$-axis ceases to grow with increasing $L$ for $L \geq 18$ as for the standard $X Y$ model while the Ising specific heat ridge continues to grow as the system size increases. From the specific heats of several lattice sizes we find that the growth rate of the specific heat ridges in the small $A$ and $C$ region is no higher than that of the Ising ridge parallel to $A$-axis which implies that the exponent $\alpha \leq 0$ for either contribution. Because of the slow increase of the Ising ridge with $L$ and the limitation to fairly small $L$, we are unable to obtain any reasonable estimate of the exponent $\alpha / \nu$.

Having established the existence of roughening and Ising transitions we can now locate the precise location of the two transition lines. It is relatively simple to locate the Ising transition line by using the probability distribution of the Ising magnetization $M_{\mathrm{I}}, P_{L}\left(M_{\mathrm{I}}, A, C\right)$. In a phase with Ising order, $P_{L}\left(M_{\mathrm{I}}=0, A, C\right)$ decreases to zero as the system size increases while $P_{L}\left(M_{\mathrm{I}}=0, A, C\right)$ increases as $L$ increases in an Ising disordered phase. We then expect that there will be a unique crossing point $\left(\mathrm{A}^{*}, \mathrm{C}^{*}\right)$ in a plot of $P_{L}\left(M_{\mathrm{I}}=0, A, C\right)$ curves for several lattice sizes along a path in the $A-C$ plane if the path crosses the transition line. Note that this does not use the concept of finite size scaling. This probability distribution method is a powerful tool to locate the transition point if the system is appropriate to this type of analysis. We have obtained $P_{L}\left(M_{\mathrm{I}}=0, A, C\right)$ curves in the $A$ - $C$ plane for lattice sizes $L=10,14,18,22$ and found that there exists a well defined line of intersection points from several $P_{L}\left(M_{\mathrm{I}}=0, A, C\right)$ surfaces which is shown in Fig. $4(\mathrm{~b})$. The estimated positional uncertainty of the Ising transition line in the $A-C$ plane is at most 0.001 . Unfortunately, it is difficult to find any RSOS order parameter suitable for this type of analysis to locate the transition line and, as an alternative method, we use the position of the peak in the susceptibility of the RSOS order parameter $M_{\mathrm{R}}$. In the rough phase, this susceptibility is infinite in the thermodynamic limit and, for finite $L$, the susceptibility peak grows as $L^{2-\eta}$. In the thermodynamic limit, the position of this diverging peak gives the transition point. 
The peak position in a finite system, for fixed $C$, approaches its bulk value $A^{*}$ as

$$
\left|A_{\text {peak }}(L)-A^{*}\right| \propto L^{-\frac{1}{\nu}}
$$

where $A_{\text {peak }}(L)$ is the peak position for lattice size $L$. In Fig. 4(a) we plot $A_{\text {peak }}(L)$ versus $L^{-1}$ for several $C=0.10,0.15,0.20$, and 0.25 from bottom to top. All points are on almost straight lines which implies that Eq. (15) describes the scaling relation of the peak position with $\nu=1$. Extrapolating to $L^{-1}=0$, we obtain the bulk value of $A^{*}$ for several fixed $C$ 's. The specific heat exponent $\alpha \leq 0$ along the roughening transition line, as we argued earlier, and this implies $\nu \geq 1$. In fact, at the roughening transition point which is the KT point in the $X Y$ model, the exponent $\nu=\infty$. If $\nu>1, A_{\text {peak }}$ is convex up in $L^{-1}$ and the extrapolated $A^{*}$ under the assumption $\nu=1$ is an upper bound for the transition point. In Fig. 4(b) we draw the roughening transition line extrapolated from Eq.(15) with $\nu=1$ together with the Ising transition line. The IOR and DOF phases are completely separated by the IOF phase in all parameter space $(A, C)$. Since the phases are separated when we use an upper bound for $A^{*}$, they will certainly be separate for the true values of $A^{*}$ as this will increase the separation. The phase trajectory of the FFXY model goes from IOR to DOF as the temperature increases from zero and the result is strong evidence for the double transition scenario in the FF $X Y$ model.

Since the two phase transition lines are accurately located some critical parameters along these lines can be estimated. We have calculated the Ising susceptibility from the usual definition and estimated the exponent $\gamma / \nu$ along the Ising transition line using sizes $L=$ $6,10,14,18,22$ for the fit (See Fig. 5). The exponent $\gamma / \nu$ is consistent with the Ising value down to $A=0.8$ but deviates significantly for smaller $A$ where the two transition lines become close. Presumably this discrepancy is due to large corrections to scaling due to large fluctuations of the RSOS order and the limited system sizes. The height-height correlation function of the RSOS model defined by $G\left(\mathbf{r}_{I}-\mathbf{r}_{J}\right) \equiv\left\langle\left(h_{I}-h_{J}\right)^{2}\right\rangle$ behaves as $G\left(\mathbf{r}_{I}-\mathbf{r}_{J}\right) \sim$ $a(A, C) \log \left(\left|\mathbf{r}_{I}-\mathbf{r}_{J}\right|\right)$ in the rough phase. The coefficient $a(A, C)$ changes continuously in the rough phase and has a universal value $2 / \pi^{2}$ at the roughening transition point. 18 The height 
width $\sigma^{2}$ is defined by $\sum_{I}\left(h_{I}-\bar{h}\right)^{2} / L^{2}$, where $\bar{h}$ is the average height and, by integrating

the height-height correlation function, $\sigma^{2} \approx \frac{a}{2} \log (L)+b$ in the rough phase. From the MC simulations, we have obtained $\sigma^{2}(A, C)$ in the IOR phase and found that the height width $\sigma^{2}$ agrees well with the $\log L$ scaling form in the whole IOR region. The coefficient $a$ increases as $A$ decreases from $A^{*}$ into the rough phase, as expected. Along the roughening transition line where two transitions are not close to each other, the coefficient $a \approx 0.16$, which is somewhat smaller than the universal value $2 / \pi^{2} \approx 0.203$. We find that the coefficient $a \approx 0.19$ along the roughening transition line where two transitions are very close to each other. We do not use any scaling or extrapolation method to locate the RSOS transition points in the region where two transitions are well separated but simply use the RSOS susceptibility peak positions of the largest lattice size, $L=22$, as the transition points. This peak position moves very slowly toward smaller $A$ as the system size becomes larger, as expected. In fact the estimated $a$ along the roughening transition line in that region decreases slightly as the system size increases. This means that the estimated transition line actually lies in the flat region close to the real roughening transition line which will lie to the left of our estimated line. We have located a more reasonable roughening transition line in that region using the criterion $a=2 / \pi^{2}$ and find that the transition line shifts to the left about $6 \%$ in $A$. This is consistent with our arguments that the true $A^{*}$ is smaller than the value estimated on the assumption that $\nu=1$ at the roughening transition and that the two transitions are separated by a thin tongue of IOF phase.

\section{CONCLUSIONS}

We have investigated the phase transitions in a restricted solid on solid model coupled to an Ising model by a rejection free microcanonical Monte Carlo method. This model is derived from the coupled $X Y$-Ising system by a duality transformation on the $X Y$ degrees of freedom and by making what appear to be reasonable restrictions on the allowed height differences. The $X Y$-Ising model is a coarse-grained generalization of the fully frus- 
trated $X Y$ model which was originally introduced in the hope that it would make numerical studies of the FFXY system easier. Despite this convoluted chain of transformations and approximations, the RSOS-Ising model is believed to undergo continuous phase transitions in the same universality classes as the FFXY system. We believe that the restriction on the heights $|\Delta h|=0,1$ is not a serious one in that it will not affect the universality class of the transitions provided they are continuous and that the topology of the phase diagram is also not qualitatively affected. We believe that the only possible difference lifting the RSOS restriction on the heights could have would be to allow the Ising and roughening lines to merge into a segment of first order transition line separating the DOF and IOR phases. In our numerical study of the RSOS-Ising system we identified three phases: Ising ordered rough (IOR), Ising ordered flat (IOF) and Ising disordered flat (DOF) which correspond to $X Y$ and Ising ordered, $X Y$ disorder and Ising order and complete disorder respectively. Using the rejection free microcanonical method, we obtain a precise phase diagram and demonstrate that the IOR and DOF phases are separated by a sliver of IOF phase for all physical values of the parameters. As the temperature in the FF $X Y$ model is raised, the system will follow some path in the $A, C$ plane from the IOR phase to the DOF phase. Since it must pass through the intervening IOF phase, this is strong evidence that there are two continuous transitions in the FF $X Y$ and $X Y$-Ising models.

The critical exponents along the Ising transition line seem to change continuously in the region where the Ising and roughening transitions are close together but this may be due to large corrections to scaling caused by the nearby roughening transition. In that case the correct determination of the critical behavior will require very large systems. Since we have located the precise phase transition lines the true critical behavior along the Ising line may be determined using other MC methods appropriate for the very large systems needed. We leave this for future study. 


\section{ACKNOWLEDGMENTS}

This work was supported in part by the NSF through Grant No. DMR-9222812 (JMK), in part by the Ministry of Education, Republic of Korea through a grant to the Research Institute for Basic Sciences, Seoul National University, in part by the Korea Science Foundation through Research Grant to the Center for Theoretical Physics, Seoul National University, and in part by SNUCTP-Brown exchange program. SL would like to thank the DaeWoo Foundation for financial support. KCL would like to thank Kyungsik Kang and many people of Brown University for the hospitality extended to him during his stay at Brown University on his last sabbatical leave. 


\section{REFERENCES}

${ }^{1}$ S. Teitel and C. Jayaprakash, Phys. Rev. B 27, 598 (1983).

${ }^{2}$ M. Y. Choi and D. Stroud, Phys. Rev. B 32, 5773 (1985).

${ }^{3}$ G. S. Grest, Phys. Rev. B 39, 9267 (1989).

${ }^{4}$ J. R. Lee, Phys. Rev. B 49, 3317 (1994); 50, 3149 (1994).

${ }^{5}$ E. Granato, J. M. Kosterlitz, and J. Lee, Phys. Rev. Lett. 66, 1090 (1991); J. Lee, E. Granato, and J. M. Kosterlitz, Phys. Rev. B 44, 4819 (1991).

${ }^{6}$ S. Lee and K.-C. Lee, Phys. Rev. B 49, 15184 (1994).

${ }^{7}$ P. Olsson, Phys. Rev. Lett. 75, 2758 (1996).

${ }^{8}$ J. M. Thijssen and H. J. F. Knops, Phys. Rev. B 42, 2438 (1990).

${ }^{9}$ D.R. Nelson and J.M. Kosterlitz, Phys. Rev. Lett. 39, 1201 (1977)

${ }^{10}$ J. M. Kosterlitz and D. J. Thouless, J. Phys. C 6, 1181 (1973).

${ }^{11}$ M. den Nijs, Phys. Rev. B 46, 10386 (1992).

${ }^{12}$ E. Carlon, Phys. Rev. E 53, R5549 (1996).

${ }^{13}$ K.-C. Lee, J. Phys. A 23, 2087 (1990); 28, 4835 (1995).

${ }^{14}$ J. José, L.P. Kadanoff, S. Kirkpatrick and D.R. Nelson, Phys. Rev. B16, 1217 (1977)

${ }^{15}$ H. Weber and P. Minnhagen, Phys. Rev. B 37, 5986 (1988).

${ }^{16}$ H.W.J. Blöte and M.P. Nightingale, Phys. Rev. B47, 15046 (1993)

${ }^{17}$ M. E. Fisher, M. N. Barber, and D. Jasnow, Phys. Rev. A 8, 1111 (1973).

${ }^{18}$ B. Nienhuis, in Phase Transitions and Critical Phenomena, edited by C. Domb and J. L. Lebowitz (Academic Press, London, 1987), Vol. 11, p1-53. 


\section{FIGURES}

FIG. 1. Representation of bond variables by integer height variables on the dual lattice. Under the height variable scheme all constraints on bond variables are automatically satisfied.

FIG. 2. (a) Surface flatness $M_{\mathrm{R}}$ of the RSOS part for lattice size $L=22$. It is easily seen that there is a roughening transition between flat $\left(M_{\mathrm{R}} \simeq 1\right)$ and $\operatorname{rough} \operatorname{phases}\left(M_{\mathrm{R}} \simeq 0.5\right)$. (b) Magnetization $M_{\mathrm{I}}$ of the Ising part for lattice size $L=22$. There is a relatively sharp transition region in the Ising magnetization. In the region of small $A$ and $C$ the Ising transition line is close to the roughening transition line. It should be noted that the viewing angle of $(\mathrm{b})$ is $90^{\circ}$ rotated from that of (a).

FIG. 3. Specific heat $C_{\mathrm{R}}$ of the RSOS part for lattice size $L=22$. The specific heat ridge parallel to $C$-axis does not increase for $L \geq 18$ just as in the $X Y$ model. (b) Specific heat $C_{\mathrm{I}}$ of the Ising part for lattice size $L=22$. As expected, the specific heat ridge grows as $L$ increases. The location of the Ising ridge is very close to that of the RSOS ridge in small $A$ and $C$ region.

FIG. 4. (a) Extrapolating the peak position of the $M_{\mathrm{R}}$ susceptibility in the bulk limit for several $C$ values. Each line corresponds to $C=0.10,0.15,0.20$ and 0.25 from bottom to top. The data points on the $A$-axis $\left(\frac{1}{L}=0\right)$ indicate extrapolated values. (b) Phase diagram of the RSOS-Ising model in the $A, C$ plane. The solid and dotted lines represent the Ising and roughening transition, respectively. The roughening transition line is obtained by extrapolations of the $M_{\mathrm{R}}$ susceptibility peak positions. For the comparison we present another phase diagram in the inset, where the roughening transition line is the position of peak in the $M_{\mathrm{R}}$ susceptibility for $L=22$.

FIG. 5. Exponent $\gamma / \nu$ of the Ising susceptibility along the Ising transition line. For $A>0.8$ $\gamma / \nu \approx 1.75$ as in the $2 \mathrm{D}$ Ising model. This value is obtained by using the best fit for the finite-size scaling relation for the susceptibility $\chi \propto L^{\gamma / \nu}$ with $L=6,10,14,18$, and 22 . 


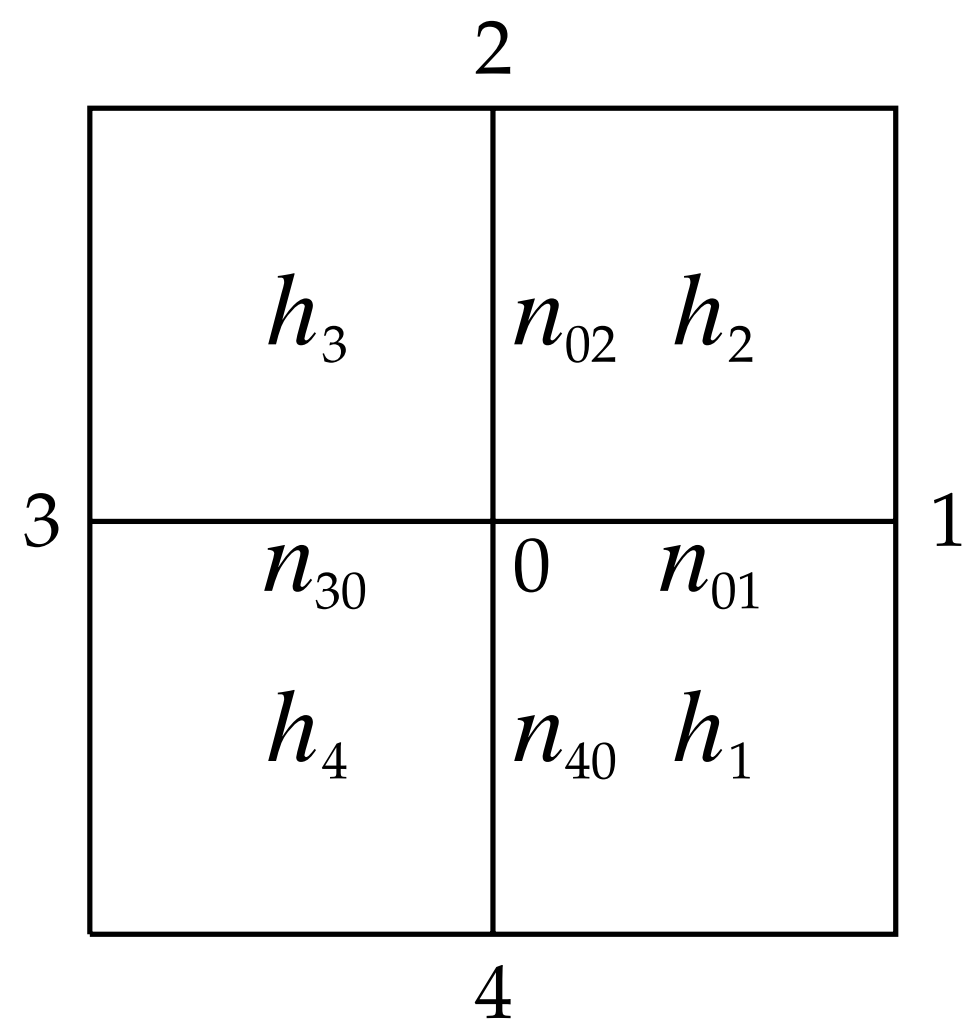

FIG. 1 


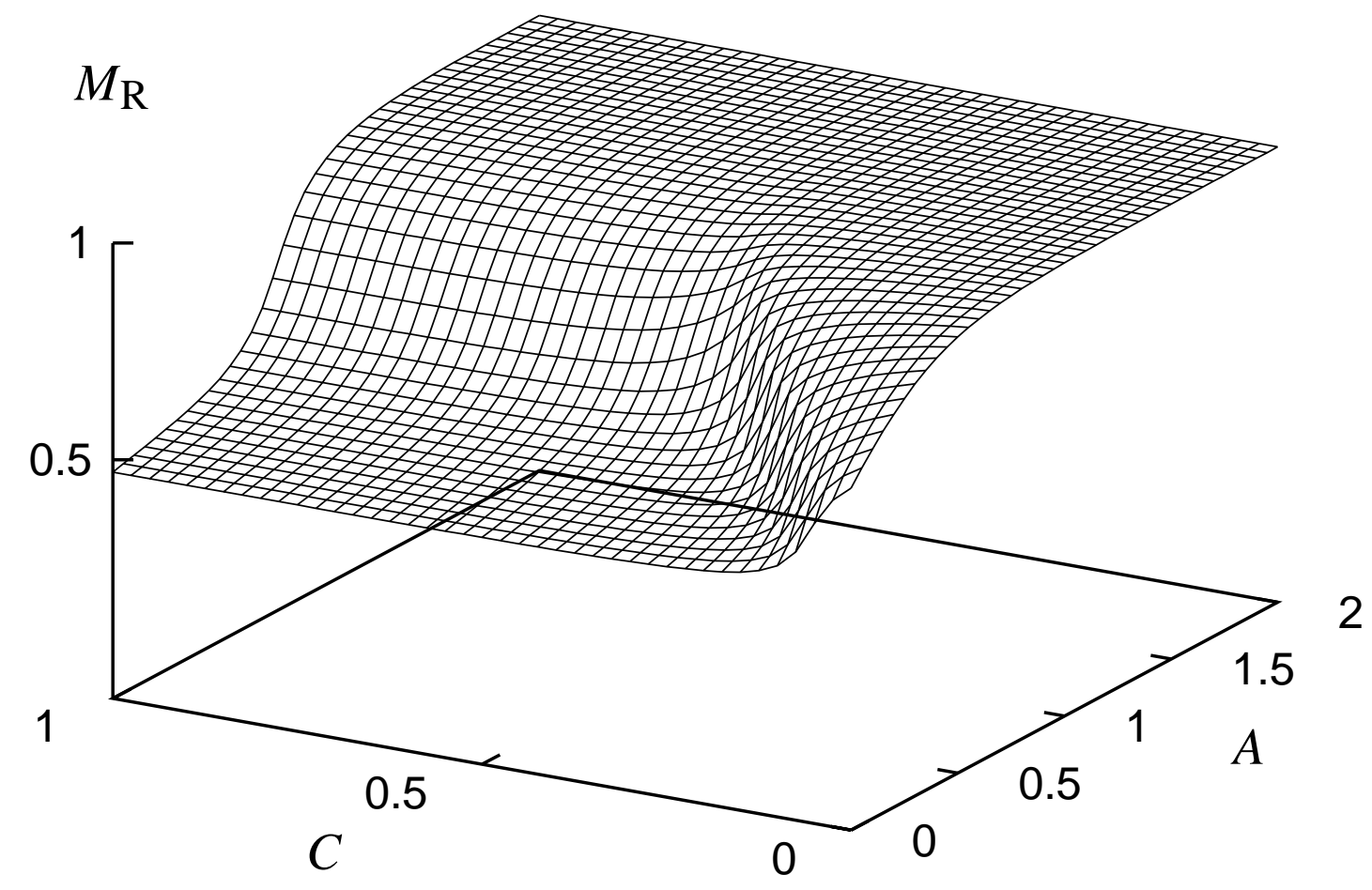

FIG. 2(a) 


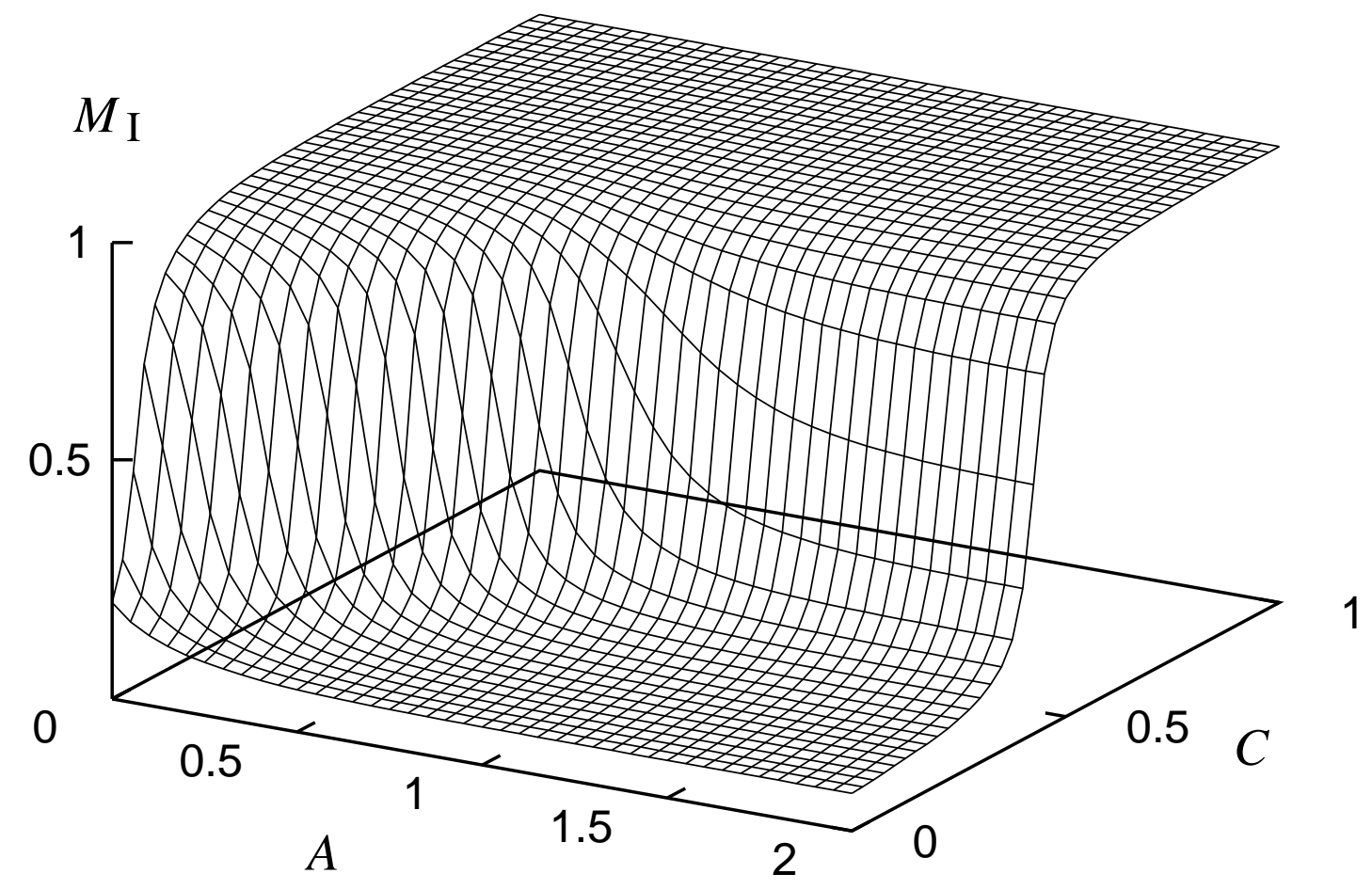

FIG. 2(b) 


\section{$C_{\mathrm{R}}$}

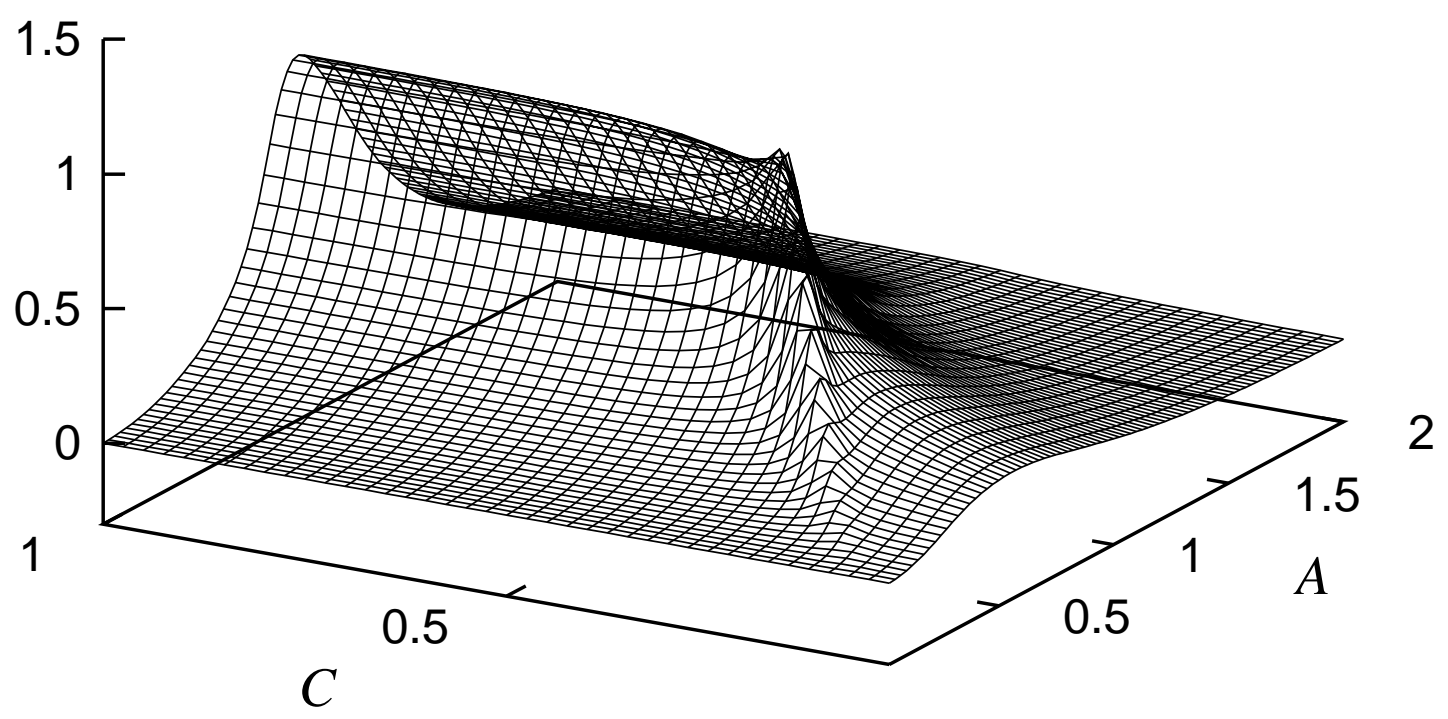

FIG. 3(a) 


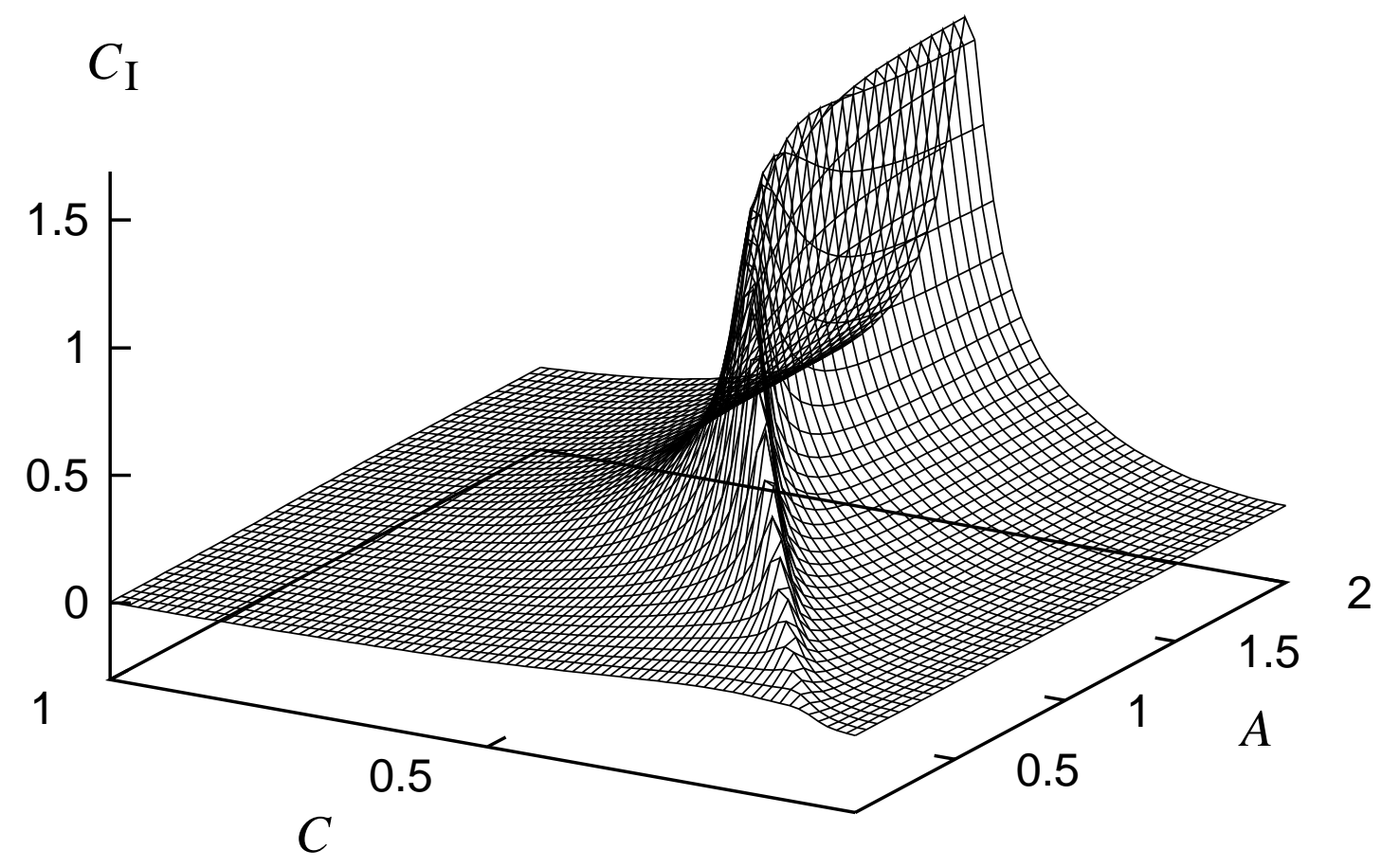

FIG. 3(b) 


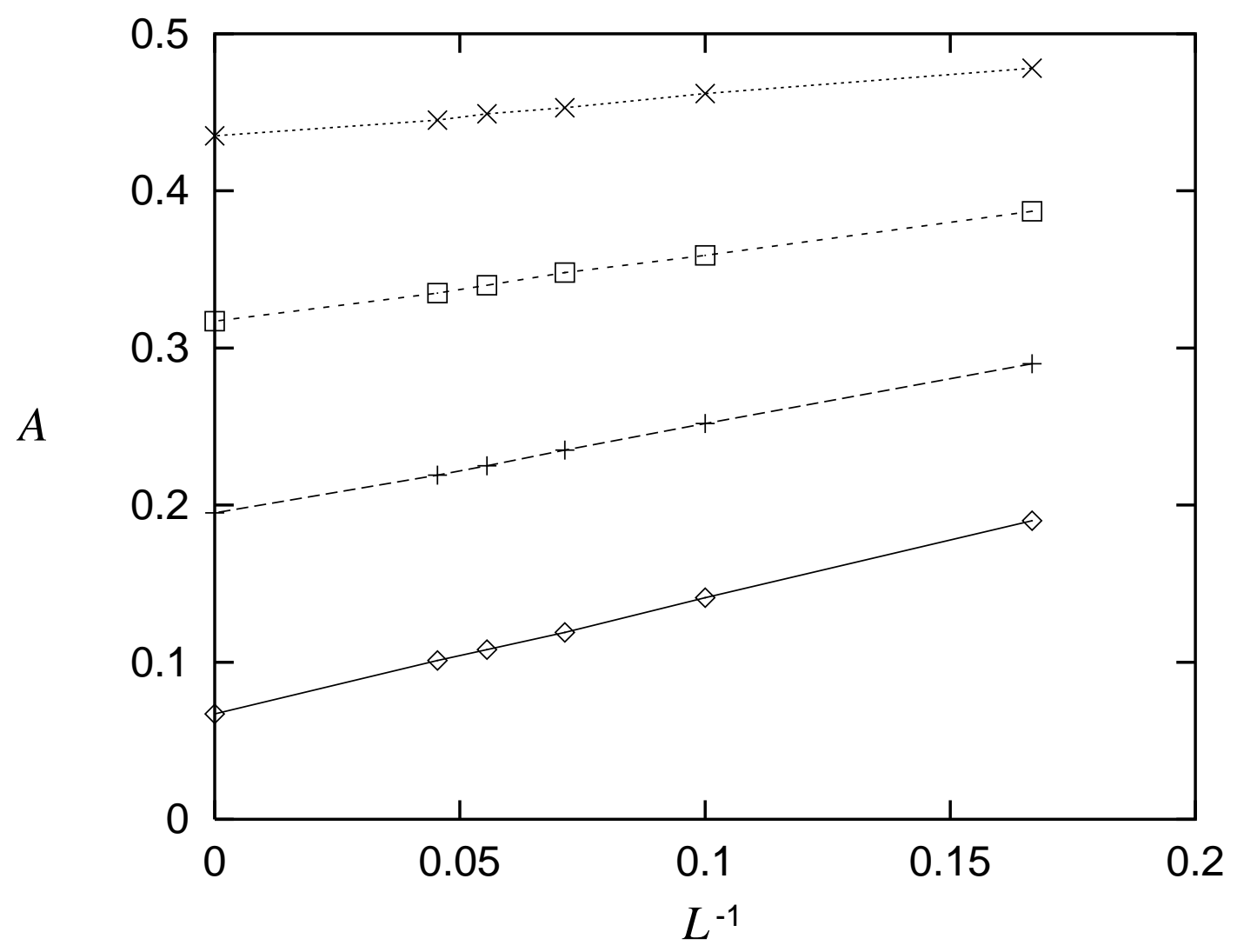

FIG. 4(a) 


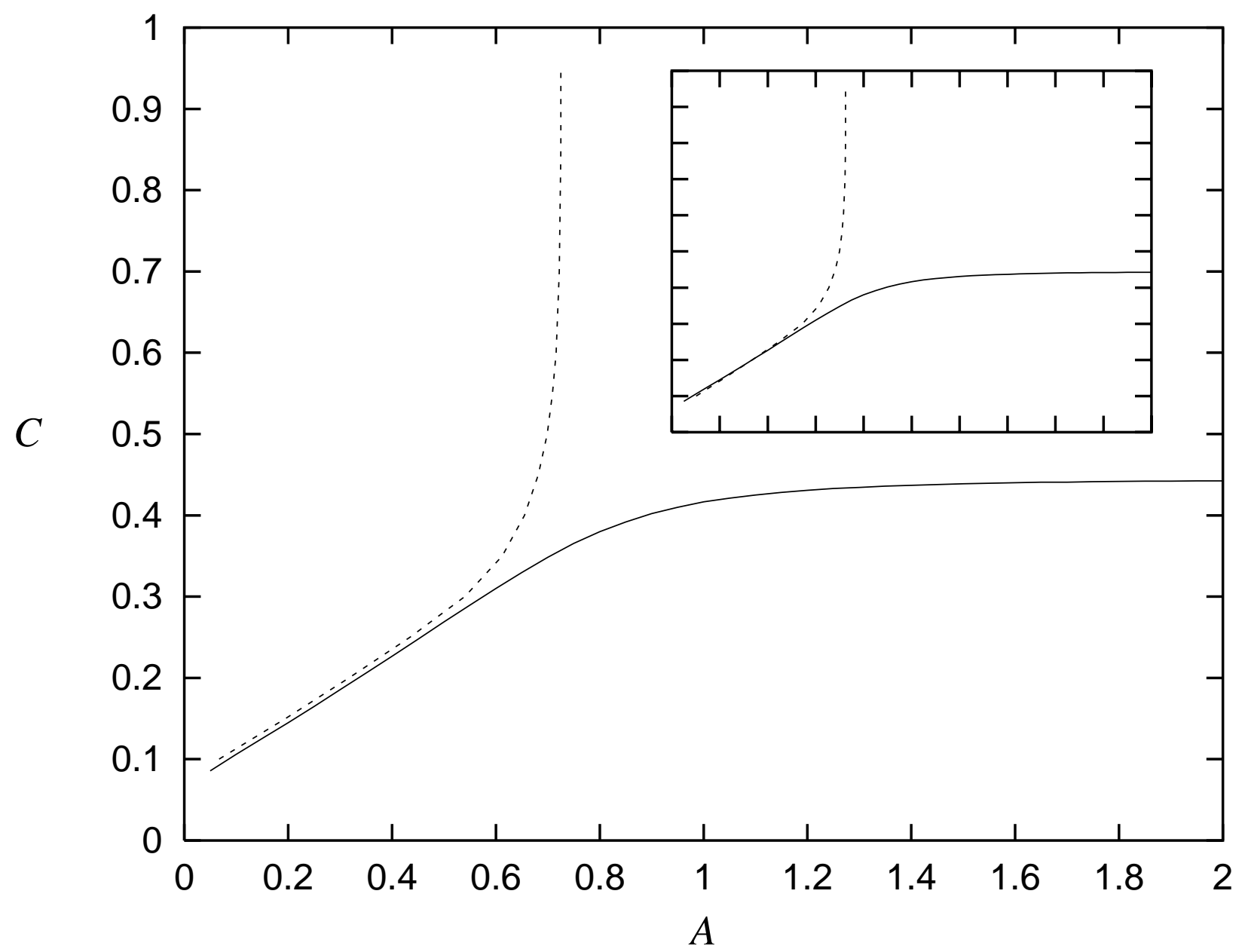

FIG. 4(b) 


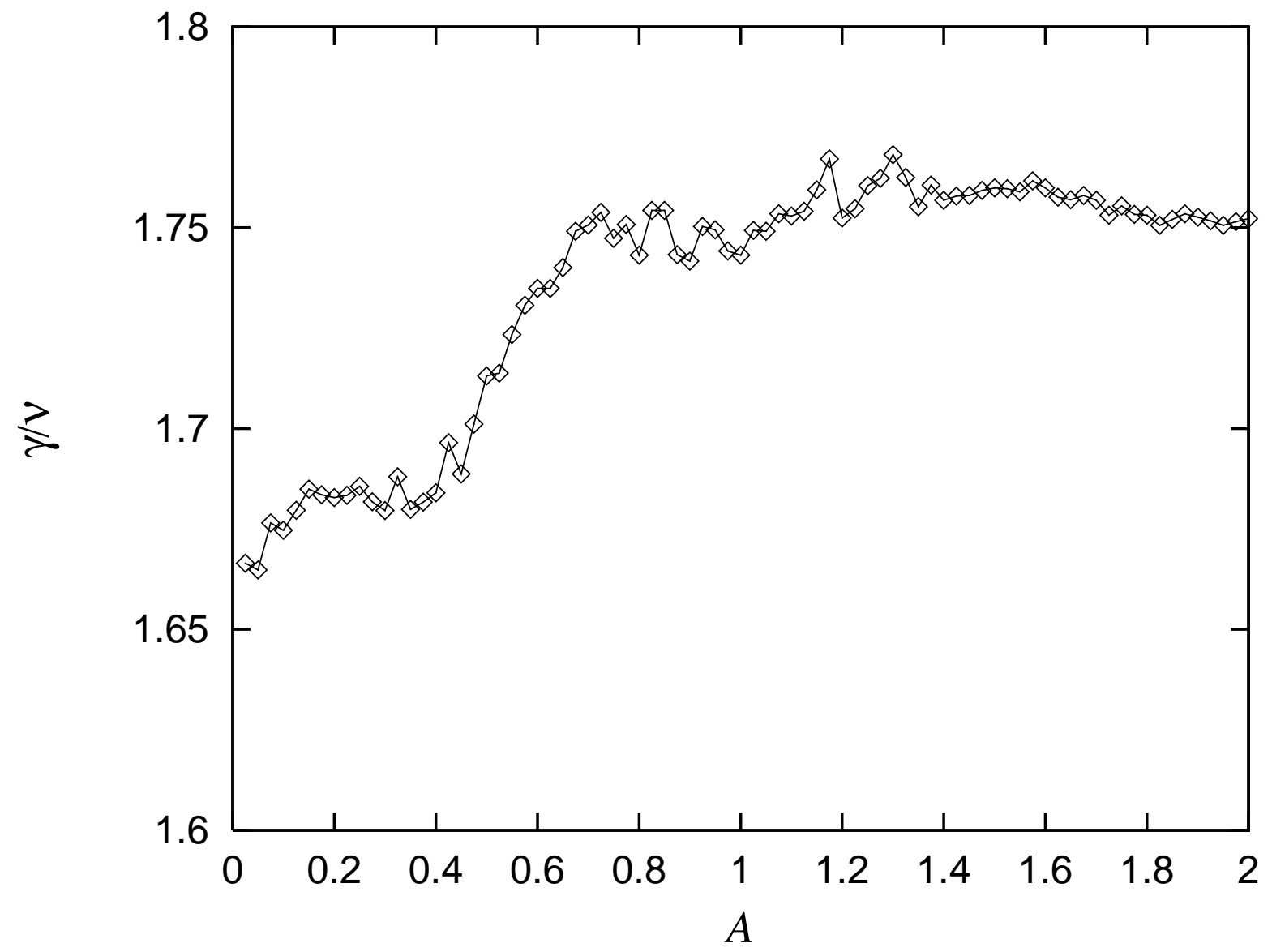

FIG. 5 